\title{
Power Series, Bieberbach Conjecture and the de Branges and Weinstein Functions
}

\author{
Wolfram Koepf \\ Department of Mathematics and Computer Science \\ University of Kassel \\ Heinrich-Plett-Str. 40 \\ D-34132 Kassel \\ Germany \\ koepf@mathematik.uni-kassel.de
}

\begin{abstract}
It is well-known that de Branges' original proof of the Bieberbach and Milin conjectures on the coefficients $a_{n}$ of univalent functions $f(z)=\sum_{k=1}^{\infty} a_{n} z^{n}$ of the unit disk as well as Weinstein's later proof deal with the same special function system that de Branges had introduced in his work.

These hypergeometric polynomials had been already studied by Askey and Gasper who had realized their positiveness. This fact was the essential tool in de Branges' proof.

In this article, we show that many identities, e.g. the representation of their generating function w.r.t. $n$, for these polynomials, which are intimately related to the Koebe function $K(z)=\sum_{k=1}^{\infty} n z^{n}$ and therefore to univalent functions, can be automatically detected from power series computations by a method developed by the author and accessible in several computer algebra systems.

In other words, in this paper a new and interesting application of the FPS (Formal Power Series) algorithm is given. As working engine we use a Maple implementation by Dominik Gruntz and the author. In particular, the hypergeometric representation of both the de Branges and the Weinstein functions are determined by successive power series computations from their generating functions.

The new idea behind this algorithm is the observation that hypergeometric function coefficients of double series can be automatically detected by an iteration of the FPS procedure.

In a final section we show how algebraic computation enables the fast verification of Askey-Gasper's positivity results for specific (not too large) $n$ using Sturm sequences or similar approaches.
\end{abstract}

\section{Categories and Subject Descriptors}

G.4 [Mathematical Software]: Algorithm design and analysis; G.2.1 [Discrete Mathematics]: Combinatorics-Generating functions, Recurrences and difference equations

Permission to make digital or hard copies of all or part of this work for personal or classroom use is granted without fee provided that copies are not made or distributed for profit or commercial advantage and that copies bear this notice and the full citation on the first page. To copy otherwise, to republish, to post on servers or to redistribute to lists, requires prior specific permission and/or a fee.

ISSAC'03, August 3-6, 2003, Philadelphia, Pennsylvania, USA.

Copyright 2003 ACM 1-58113-641-2/03/0008 ...\$5.00.

\section{General Terms}

Algorithms

\section{Keywords}

Bieberbach conjecture, Askey-Gasper inequality, de Branges functions, Weinstein functions, FPS algorithm, algorithmic computation of formal power series, Sturm sequences.

\section{INTRODUCTION}

Let $S$ denote the family of analytic and univalent functions $f(z)=z+a_{2} z^{2}+\ldots$ of the unit disk $\mathbb{D}$. $S$ is compact with respect to the topology of locally uniform convergence so that $k_{n}:=\max _{f \in S}\left|a_{n}(f)\right|$ exists. In 1916 Bieberbach [2] proved that $k_{2}=2$, with equality if and only if $f$ is a rotation of the Koebe function

$$
K(z):=\frac{z}{(1-z)^{2}}=\frac{1}{4}\left(\left(\frac{1+z}{1-z}\right)^{2}-1\right)=\sum_{n=0}^{\infty} n z^{n},
$$

and in a footnote he mentioned "Vielleicht ist überhaupt $k_{n}=n . "{ }^{1}$ This statement is known as the Bieberbach conjecture.

In 1923 Löwner [14] proved the Bieberbach conjecture for $n=3$. His method was to embed a univalent function $f(z)$ into a Löwner chain, i.e. a family $\{f(z, t) \mid t \geqq 0\}$ of univalent functions of the form

$f(z, t)=e^{t} z+\sum_{n=2}^{\infty} a_{n}(t) z^{n}, \quad\left(z \in \mathbb{D}, t \geqq 0, a_{n}(t) \in \mathbb{C}(n \geqq 2)\right)$

which start with $f$

$$
f(z, 0)=f(z)
$$

and for which the relation

$$
\operatorname{Re} p(z, t)=\operatorname{Re}\left(\frac{\dot{f}(z, t)}{z f^{\prime}(z, t)}\right)>0 \quad(z \in \mathbb{D})
$$

is satisfied. Here' and 'denote the partial derivatives with respect to $z$ and $t$, respectively. Equation (2) is referred to as the Löwner differential equation, and geometrically it states that the image domains of $f(\mathbb{D}, t)$ expand as $t$ increases.

\footnotetext{
${ }^{1}$ Perhaps $k_{n}=n$ is generally valid.
} 
The history of the Bieberbach conjecture showed that it was easier to obtain results about the logarithmic coefficients of a univalent function $f$, i.e. the coefficients $d_{n}$ of the expansion

$$
\varphi(z)=\ln \frac{f(z)}{z}=: \sum_{n=1}^{\infty} d_{n} z^{n}
$$

rather than for the coefficients $a_{n}$ of $f$ itself. So Lebedev and Milin [13] in the mid sixties of the last century developed methods to exponentiate such information. They proved that if for $f \in S$ the Milin conjecture

$$
\sum_{k=1}^{n}(n+1-k)\left(k\left|d_{k}\right|^{2}-\frac{4}{k}\right) \leqq 0
$$

on its logarithmic coefficients is satisfied for some $n \in \mathbb{N}$, then the Bieberbach conjecture for the index $n+1$ follows.

In 1984 de Branges [3] verified the Milin, and therefore the Bieberbach conjecture, and in 1991, Weinstein [16] gave a different proof. Both proofs use special function systems, and independently, Todorov [15] and Wilf [17] discovered that these essentially are the same, see also [11], [12].

In [5], Zeilberger showed how parts of Weinstein's proof version can be principally computerized. The main argument is the following: The coefficients $C_{k, n}(x)$ of the function

$$
\begin{gathered}
\frac{1}{\sqrt{1-z\left(2 x^{2}+\left(1-z^{2}\right)(w+1 / w)\right)+z^{2}}}= \\
\sum_{n=0}^{\infty} \sum_{k=0}^{n} \frac{(n-k) !}{(n+k) !}\left(1-x^{2}\right)^{k} C_{k, n}(x)\left(w^{k}+w^{-k}\right) z^{n}
\end{gathered}
$$

are polynomials $C_{k, n}(x) \in \mathbb{Q}[x]$ with rational coefficients. To prove that these form the squares of another system of polynomials $D_{k, n} \in \mathbb{Q}[x]$,

$$
D_{k, n}(x)^{2}=C_{k, n}(x)
$$

Zeilberger suggests to calculate the first polynomials $D_{k, n}(x)$ for $0 \leq k \leq n \leq 20$, then to "guess" a holonomic recurrence equation w.r.t. $n$

$$
\sigma_{2} D_{k, n+2}(x)+\sigma_{1} D_{k, n+1}(x)+\sigma_{0} D_{k, n}(x)=0
$$

with polynomials $\sigma_{j} \in \mathbb{Q}[k, n]$ of fixed degrees w.r.t. $k$ and $n$, and to use linear algebra to find $\sigma_{0}, \sigma_{1}, \sigma_{2}$. In a final part, the initial guess can be a posteriori verified by the WZ method [18] finishing this part of Weinstein's proof. ${ }^{2}$ Note, however, that this approach is much different from the approach of the given paper which mechanizes other parts of the given proofs.

In this article, we show that many identities, e.g. the representation of their generating function, for the de Branges and Weinstein polynomials, which are intimately related to the Koebe function and therefore to univalent function theory, can be automatically detected from power series computations by a method developed by the author and accessible in several computer algebra systems.

\footnotetext{
${ }^{2}$ Although the ideas of the article [5] are very interesting, the presentation of some details is not completely correct. Hence we refer the reader to my review 0894.30013 in Zentralblatt für Mathematik which contains a corrected version which was approved by Zeilberger.
}

As working engine we use a Maple implementation by Dominik Gruntz and the author. In particular, the hypergeometric representations of the de Branges and Weinstein functions are determined by successive power series computations from their generating functions.

\section{AUTOMATIC COMPUTATION OF POWER SERIES COEFFICIENTS}

In this section, we review the FPS algorithm enabling the automatic computation of power series as designed in [8].

Given an expression $f(x)$ in the variable $x$, one would like to find the Taylor series

$$
f(x)=\sum_{k=0}^{\infty} A_{k} x^{k},
$$

i.e., a formula for the coefficient $A_{k}$. For example, if $f(x)=$ $\exp (x)$, then

$$
f(x)=\sum_{k=0}^{\infty} \frac{1}{k !} x^{k}
$$

hence $A_{k}=\frac{1}{k !}$.

If the result is simple enough, the FPS (formal power series) procedure of the Maple package FPS.mpl ([8], [6], [7]) computes this series, even if it is a Laurent series (including negative powers) or Puiseux series (including rational powers).

The main idea behind this procedure is

1. to compute a holonomic differential equation for $f(x)$, i.e., a homogeneous linear differential equation with polynomial coefficients,

2. to convert the differential equation to a holonomic recurrence equation for $A_{k}$,

3. and to solve the recurrence equation for $A_{k}$.

The above procedure is successful at least is $f(x)$ is hypergeometric. A series

$$
\sum_{k=0}^{\infty} a_{k}
$$

is called hypergeometric, if the series coefficients $a_{k}$ have rational term ratio

$$
\frac{a_{k+1}}{a_{k}} \in \mathbb{C}(k)
$$

The function

$$
\begin{gathered}
{ }_{p} F_{q}\left(\begin{array}{c}
a_{1}, a_{2}, \ldots, a_{p} \\
b_{1}, b_{2}, \ldots, b_{q}
\end{array} \mid x\right):= \\
\sum_{k=0}^{\infty} A_{k} x^{k}=\sum_{k=0}^{\infty} \frac{\left(a_{1}\right)_{k} \cdot\left(a_{2}\right)_{k} \cdots\left(a_{p}\right)_{k}}{\left(b_{1}\right)_{k} \cdot\left(b_{2}\right)_{k} \cdots\left(b_{q}\right)_{k}} \frac{x^{k}}{k !}
\end{gathered}
$$

is called the generalized hypergeometric series, since its term ratio

$$
\frac{A_{k+1} x^{k+1}}{A_{k} x^{k}}=\frac{\left(k+a_{1}\right) \cdots\left(k+a_{p}\right)}{\left(k+b_{1}\right) \cdots\left(k+b_{q}\right)} \frac{x}{(k+1)}
$$

is a general rational function, in factorized form. Here $(a)_{k}=$ $a(a+1) \cdots(a+k-1)$ denotes the Pochhammer symbol or shifted factorial. The summand $a_{k}$ of the generalized hypergeometric series is called a hypergeometric term. 


\section{THE LÖWNER CHAIN OF THE KOEBE FUNCTION}

In this section, we consider the Löwner chain

$$
W(z, t):=K^{-1}\left(e^{-t} K(z)\right) \quad(z \in \mathbb{D}, t \geqq 0)
$$

of bounded univalent functions in the unit disk $\mathbb{D}$ which is defined in terms of the Koebe function (1). Since $K$ maps the unit disk onto the entire plane slit along the negative $x$ axis in the interval $(-\infty, 1 / 4], W(\mathbb{D}, t)$ is the unit disk with a radial slit increasing with $t$.

A computation shows that

$$
W(z, t)=\frac{4 e^{-t} z}{\left(1-z+\sqrt{1-2\left(1-2 e^{-t}\right) z+z^{2}}\right)^{2}}
$$

(see [11], after Eq. (47)).

As a first application of the FPS algorithm, we compute the Taylor series of $w(z, y)=W(z,-\ln y)$, considered as function of the variable $y:=e^{-t}$,

$$
w(z, y)=\frac{4 y z}{\left(1-z+\sqrt{1-2(1-2 y) z+z^{2}}\right)^{2}},
$$

which turns out to be hypergeometric:

$$
\begin{gathered}
>\quad \text { read "FPS.mpl"; } \\
\text { Package Formal Power Series, Maple V-8 } \\
\text { Copyright 1995, Dominik Gruntz, University of Basel } \\
\text { Copyright 2002, Detlef Müller \& Wolfram Koepf, } \\
\text { University of Kassel } \\
>\quad \text { assume }(\mathbf{z}<1, \mathbf{z}>0) \text {; interface (showassumed=0); } \\
>\quad \mathrm{w}:=4 * \mathrm{y} * \mathrm{z} /\left(1-\mathrm{z}+\mathrm{sqrt}\left(1-2 *(1-2 * \mathrm{y}) * \mathbf{z}+\mathrm{z}^{\wedge} 2\right)\right)^{\wedge} 2: \\
>\quad \mathrm{s}:=\text { standardsum(FPS }(\mathrm{w}, \mathrm{y}, \mathrm{j})) ; \\
s:=\sum_{j=0}^{\infty}\left(\frac{2(-1)^{j}\left(\frac{1}{(z-1)^{2}}\right)^{j}(1+2 j) ! z^{(j+1)} y^{(j+1)}}{(z-1)^{2}(j !)^{2}(j+1)(j+2)}\right)
\end{gathered}
$$

Note that standardsum puts the sum signs in front of the expression.

Essentially by computing and simplifying the coefficient term ratio, from the above result one gets the hypergeometric representation for $w(z, y)([9],[10] \text {, Chapter } 2)^{3}$

$$
\begin{aligned}
& >\text { factor(sumtools [Sumtohyper] }(\mathrm{op}(1, \mathrm{~s}), \mathrm{j})) \text {; } \\
& -\frac{1}{2} \frac{(z-1)^{2} \text { Hypergeom }\left(\left[\frac{-1}{2}\right],[],-\frac{4 z y}{(z-1)^{2}}\right)}{z y}
\end{aligned}
$$

To obtain this representation, as an intermediate result FPS uses the differential equation

$$
\begin{gathered}
>\mathrm{DE}:=\text { HolonomicDE }(\mathrm{w}, \mathrm{W}(\mathrm{y})) \\
D E:=y^{2}\left(1-2 z+4 z y+z^{2}\right)\left(\frac{d^{2}}{d y^{2}} \mathrm{~W}(y)\right)- \\
(-1+z)^{2} \mathrm{~W}(y)+\left(6 z y+1+z^{2}-2 z\right) y\left(\frac{d}{d y} \mathrm{~W}(y)\right)=0
\end{gathered}
$$

for $w(z, y)$.

\footnotetext{
${ }^{3}$ Sumtohyper is the inert form of sumtohyper which outputs the inert form Hypergeom for the hypergeometric series to prevent evaluation.
}

Note that the above series result can be used to find a hypergeometric representation for the Taylor coefficients $A_{n}(y)$ of the function

$$
w(z, y)=\sum_{n=0}^{\infty} A_{n}(y) z^{n}
$$

Writing

$$
A_{n}(y)=\sum_{j=0}^{\infty} a_{j}^{(n)} y^{j}
$$

one arrives at the double sum

$$
w(z, y)=\sum_{n=0}^{\infty} \sum_{j=0}^{\infty} a_{j}^{(n)} y^{j} z^{n} .
$$

By another application of the FPS algorithm, the coefficients $a_{j}^{(n)}$ of this double series can be determined, which therefore yields a representation for $A_{n}(y)$. We compute

$>$ ss:=standardsum(FPS $(\mathrm{s}, \mathrm{z}, \mathrm{i}))$;

$s s:=\sum_{j=0}^{\infty}\left(\sum_{i=0}^{\infty}\left(\frac{2(-1)^{j}(2 j) !(2 j+1) y^{j} y(2 j+2)_{i} z^{(j+i+1)}}{(j !)^{2}(j+1)(j+2) i !}\right)\right)$

By (4), the exponent of $z$ is $n$, hence we substitute $i$ by $n-j-1$

$>\operatorname{summand}:=\operatorname{subs}(i=n-j-1, o p([1,1], \mathrm{ss}))$;

summand $:=\frac{2(-1)^{j}(2 j) !(2 j+1) y^{j} y(2 j+2)_{n-j-1} z^{n}}{(j !)^{2}(j+1)(j+2)(n-j-1) !}$

to get the hypergeometric representation for $A_{n}(y)$

$>$ sumtools [sumtohyper] (summand, $j$ ); y $n z^{n}$ hypergeom $([n+1,1-n],[3], y)$

Hence our computations have generated the result

$$
\begin{gathered}
A_{n}(y)=\sum_{j=0}^{\infty} \frac{2(-1)^{j}(2 j) !(2 j+1)(2 j+2)_{n-j-1}}{(j !)^{2}(j+1)(j+2)(n-j-1) !} y^{j+1} \\
\quad=n y_{2} F_{1}\left(\begin{array}{c}
n+1,1-n \\
3
\end{array} \mid y\right)
\end{gathered}
$$

in agreement with [11], Eq. (19).

The above method can be summarized in

Theorem 1. Assume $w(z, y)$, given as function in $z$ and $y$, can be written as double sum (4) with coefficients $a_{j}^{(n)}$ that are hypergeometric terms w.r.t. both summation variables $j$ and $n$. Then a double application of the FPS algorithm, applied to $w(z, y)$, computes $a_{j}^{(n)}$, and hence gives the hypergeometric representation

$$
A_{n}(y)=\sum_{j=0}^{\infty} a_{j}^{(n)} y^{j}
$$

for the coefficient functions $A_{n}(y)$ that are generated by $w(z, y)$, i.e.

$$
w(z, y)=\sum_{n=0}^{\infty} A_{n}(y) z^{n} .
$$

We would like to mention how efficiently the above result was obtained compared to the lengthy and complicated deduction given in [11]. This statement is valid for all results of this paper. 


\section{THE DE BRANGES AND WEINSTEIN FUNCTIONS}

In [3] de Branges showed that the Milin conjecture is valid if for all $n \geqq 2$ the de Branges functions $\tau_{k}^{n}: \mathbb{R}_{\geq 0} \rightarrow \mathbb{R}(k=$ $1, \ldots, n)$ defined by the system of differential equations

$$
\tau_{k+1}^{n}(t)-\tau_{k}^{n}(t)=\frac{\dot{\tau}_{k}^{n}(t)}{k}+\frac{\dot{\tau}_{k+1}^{n}(t)}{k+1} \quad(k=1, \ldots, n)
$$

with the initial values

$$
\tau_{k}^{n}(0)=n+1-k
$$

have the properties

$$
\lim _{t \rightarrow \infty} \tau_{k}^{n}(t)=0
$$

and

$$
\dot{\tau}_{k}^{n}(t) \leqq 0 \quad\left(t \in \mathbb{R}_{\geqq 0}\right) .
$$

Whereas the relation (5) is easily checked using standard methods for ordinary differential equations, (6) is a deep result.

L. de Branges gave an explicit representation of the function system $\tau_{k}^{n}(t)[3]$ with which the proof of the de Branges theorem was completed as soon as de Branges realized that (6) was a theorem previously proved by Askey and Gasper [1].

On the other hand, Weinstein [16] uses the Löwner chain (3), and shows the validity of Milin's conjecture if for all $n \geqq 2$ the Weinstein functions $\Lambda_{k}^{n}: \mathbb{R}_{\geqq 0} \rightarrow \mathbb{R}(k=1, \ldots, n)$ defined by

$$
\frac{e^{t} W(z, t)^{k+1}}{1-W^{2}(z, t)}=: \sum_{n=k}^{\infty} \Lambda_{k}^{n}(t) z^{n+1}=L_{k}(z, t)
$$

satisfy the relations

$$
\Lambda_{k}^{n}(t) \geqq 0 \quad\left(t \in \mathbb{R}_{\geqq 0}, \quad k, n \in \mathbb{N}\right) .
$$

Weinstein did not identify the functions $\Lambda_{k}^{n}(t)$, but was able to prove (8) without an explicit representation.

Independently, both Todorov [15] and Wilf [17] provedusing the explicit representation of the de Branges functions to be developed in Section 6-that for all $n \in \mathbb{N}$, $k=1, \ldots, n$, one has the identity

$$
\dot{\tau}_{k}^{n}(t)=-k \Lambda_{k}^{n}(t),
$$

i.e. the de Branges and the Weinstein functions essentially are the same, and the main inequalities (6) and (8) are identical. Note that in [11] an algebraic proof of (9) was given which does not use the explicit representation of the de Branges functions.

\section{CLOSED FORM REPRESENTATION OF THE WEINSTEIN FUNCTIONS}

In this section, we use Theorem 1 to generate the hypergeometric representation for the Weinstein functions $\Lambda_{k}^{n}(t)$ directly from their defining generating function (7).

To generate a power series w.r.t. $y$ for the generating function of the Weinstein functions

$$
\frac{1}{y} \cdot \frac{w(z, y)^{k+1}}{1-w(z, y)^{2}}=\sum_{j=0}^{\infty} \alpha_{j} y^{j}
$$

which starts for $j=0$, we put the factor $y^{k+1}$ in front of FPS and compute

$$
\begin{aligned}
& >\quad \mathrm{s}:=\text { standardsum }\left(\mathrm{y}^{\wedge}(\mathrm{k}+1) *\right. \\
& \left.>\quad \operatorname{FPS}\left(1 / \mathrm{y} *(\mathrm{w} / \mathrm{y})^{\wedge}(\mathrm{k}+1) /\left(1-\mathrm{w}^{\wedge} 2\right), \mathrm{y}, \mathrm{j}\right)\right) ;
\end{aligned}
$$$$
s:=\sum_{j=0}^{\infty} \frac{y^{k} y(-1)^{j} z^{(j+1+k)}(1+2 k)_{2 j}\left(\frac{1}{(z-1)^{2}}\right)^{(k+j)} y^{(j-1)}}{(z-1)^{2}(1+2 k)_{j} j !}
$$

Note that the differential equation w.r.t. $y$

$$
>\mathrm{DE} 1:=\text { HolonomicDE }\left(1 / \mathrm{y} * \mathrm{w}^{\wedge}(\mathrm{k}+1) /\left(1-\mathrm{w}^{\wedge} 2\right), \mathrm{L}(\mathrm{y})\right) \text {; }
$$

$$
\begin{aligned}
& D E 1:=\left(2 z y-k^{2}+2 z k^{2}-z^{2} k^{2}\right) L(y) \\
& +y\left(10 z y+1+z^{2}-2 z\right)\left(\frac{d}{d y} L(y)\right) \\
& +y^{2}\left(1-2 z+4 z y+z^{2}\right)\left(\frac{d^{2}}{d y^{2}} L(y)\right)=0
\end{aligned}
$$

for the generating function $L_{k}(z, t)$ leads to the above hypergeometric result.

An iterated power series computation w.r.t. $z$ gives

$$
\begin{aligned}
& >\quad \text { ss }:=\operatorname{FPS}(\mathrm{s}, \mathrm{z}, \mathrm{i}) ; \\
& s s:=\sum_{j=0}^{\infty}\left(\sum_{i=0}^{\infty}(\right. \\
& \frac{2(-1)^{j} z^{(i+1+k+j)} \Gamma(2 j+2 k) y^{(k+j)}(2 j+2+2 k)_{i} j}{j !(j+2 k) \Gamma(j+2 k) i !} \\
& \left.\left.+\frac{2(-1)^{j} z^{(i+1+k+j)} y^{(k+j)} k(2 j+2+2 k)_{i} \Gamma(2 j+2 k)}{\Gamma(j+2 k)(j+2 k) j ! i !}\right)\right)
\end{aligned}
$$

with summand

$>$ summand: $=o p([1,1]$, ss $)$ :

$>$ summand:=simplify $(\operatorname{subs}(i=n-j-k$, summand $))$;

summand $:=\frac{(-1)^{j} z^{(n+1)} y^{(k+j)} \Gamma(j+2+k+n)}{(2 j+1+2 k) \Gamma(j+1+2 k) \Gamma(j+1) \Gamma(n-j-k+1)}$

This finally yields the hypergeometric representation for $\Lambda_{k}^{n}$

$$
\begin{aligned}
& \quad>\text { result } 1:=\operatorname{convert}( \\
& \quad \text { sumtools }[\text { sumtohyper] (summand, } \mathrm{j}), \text { binomial); } \\
& \text { result1 }:=z^{(n+1)} y^{k} \operatorname{binomial}(k+n+1,1+2 k) \\
& \text { hypergeom }\left(\left[\frac{1}{2}+k, k-n, 2+k+n\right],\left[1+2 k, \frac{3}{2}+k\right], y\right) \\
& \text { showing that }
\end{aligned}
$$

$$
\Lambda_{k}^{n}(z, t)=e^{-k t}\left(\begin{array}{c}
k+n+1 \\
1+2 k
\end{array}\right){ }_{3} F_{2}\left(\begin{array}{c}
\frac{1}{2}+k, k-n, 2+k+n \\
1+2 k, \frac{3}{2}+k
\end{array} \mid e^{-t}\right)
$$

see e.g. [11], Section 5. The plot

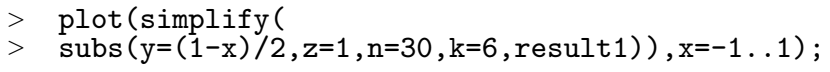




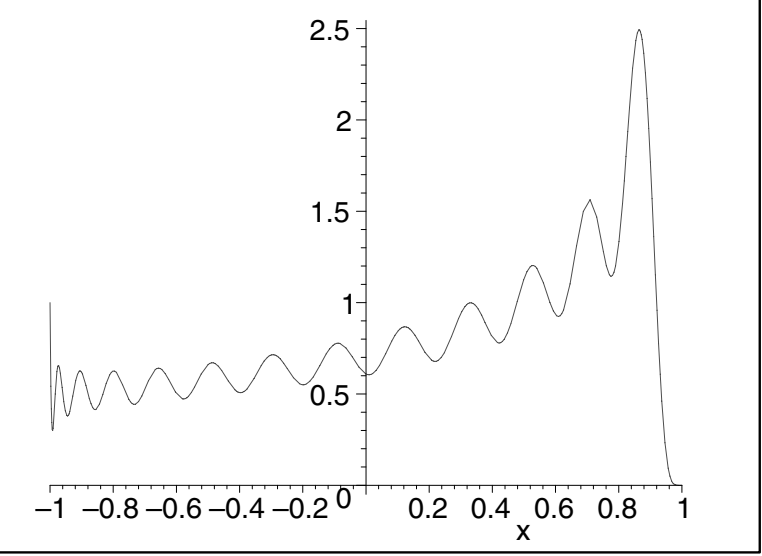

of $\Lambda_{6}^{30}$ with $y=\frac{1-x}{2}$ for $x=-1, \ldots, 1$ shows the nonnegativity of this polynomial. Moreover the highly oscillatory character of the Weinstein functions, resp. the derivatives of the de Branges functions, can be seen.

\section{GENERATING FUNCTION OF THE DE BRANGES FUNCTIONS}

In [11], a very simple generation function for the de Branges function was given:

$$
\begin{aligned}
& B_{k}(z, t)=\sum_{n=k}^{\infty} \tau_{k}^{n}(t) z^{n+1}=K(z) W(z, t)^{k} \\
= & K(z)^{k+1} e^{-k t}{ }_{2} F_{1}\left(\begin{array}{c|c}
k, k+1 / 2 \\
2 k+1
\end{array}\right. \\
= & \sum_{j=k}^{\infty}(-1)^{j+k} \frac{2 k}{j+k}\left(\begin{array}{c}
2 j-1 \\
j-k
\end{array}\right) K(z)^{j+1} e^{-j t} .
\end{aligned}
$$

Equation (11) shows the intimate relation of the de Branges functions with the Koebe function: Their generating function is basically a power of the Löwner chain of the Koebe function.

By Theorem 1 we can obtain these representations automatically. Note that these results include a hypergeometric representation of the $k$-th power of $w(z, y)$, extending the result for $k=1$ given in Section 3 .

We compute for $K(z) w(z, y)^{k}$

$>\mathrm{s}:=\operatorname{standardsum}\left(\mathrm{y}^{\wedge} \mathrm{k} * \mathrm{FPS}\left(\mathrm{z} /(1-\mathrm{z})^{\wedge} 2 *(\mathrm{w} / \mathrm{y})^{\wedge} \mathrm{k}, \mathrm{y}, \mathrm{j}\right)\right)$;

$$
s:=\sum_{j=0}^{\infty} \frac{y^{k}(-1)^{j} z^{(j+1+k)}\left(\frac{1}{(-1+z)^{2}}\right)^{(k+j)}(2 k)_{2 j} y^{j}}{(-1+z)^{2}(1+2 k)_{j} j !}
$$

and therefore get (13), which by the calculation

$>$ sumtools [Sumtohyper] $(o p(1, s), j)$;

$y^{k} z^{(k+1)}\left(\frac{1}{(-1+z)^{2}}\right)^{k}$ Hypergeom $\left(\left[k, \frac{1}{2}+k\right],[1+2 k],-\frac{4 z y}{(-1+z)^{2}}\right)$

$$
(-1+z)^{2}
$$

gives the hypergeometric representation (12) of $K(z) w(z, y)^{k}$. As intermediate result, the differential equation

$>\mathrm{DE}:=$ HolonomicDE $\left(z /(1-z)^{\wedge} 2 * \mathrm{w}^{\wedge} \mathrm{k}, \mathrm{B}(\mathrm{y})\right)$;

$$
\begin{aligned}
& D E:=\left(1-2 z+4 z y+z^{2}\right) y^{2}\left(\frac{d^{2}}{d y^{2}} \mathrm{~B}(y)\right)-k^{2}(-1+z)^{2} \mathrm{~B}(y) \\
& +y\left(6 z y+1-2 z+z^{2}\right)\left(\frac{d}{d y} \mathrm{~B}(y)\right)=0
\end{aligned}
$$

for $w(z, y)^{k}$ is used.

At this point we would like to mention that this differential equation can also be automatically determined applying a version of Zeilberger's algorithm (see e.g. [10], Chapter 7 ) to the summand of the hypergeometric representation of $w(z, y)$ :

$>$ read "hsum6.mpl";

Package "Hypergeometric Summation", Maple V-8 Copyright 1998-2002, Wolfram Koepf, University of Kassel $>$ DE2:=sumdiffeq $(o p(1, s), j, B(y))$;

$$
\begin{aligned}
& \text { DE2 }:=-\left(1-2 z+4 z y+z^{2}\right) y^{2}\left(\frac{d^{2}}{d y^{2}} \mathrm{~B}(y)\right) \\
& -y\left(z^{2}+1+6 z y-2 z\right)\left(\frac{d}{d y} \mathrm{~B}(y)\right)+k^{2}(-1+z)^{2} \mathrm{~B}(y)=0
\end{aligned}
$$

By (11), a second application of the FPS procedure leads to a hypergeometric representation of $\tau_{k}^{n}$ :

$>$ ss:=standardsum(FPS $(s, z, i))$;

ss $:=\sum_{j=0}^{\infty}$

$$
\begin{aligned}
& \left(\sum_{i=0}^{\infty}\left(\frac{2(-1)^{j} y^{k} y^{j} k \Gamma(2 j+2 k)(2 j+2+2 k)_{i} z^{(j+i+1+k)}}{\Gamma(j+2 k)(j+2 k) j ! i !}\right)\right) \\
& >\text { summand: }=o p([1,1], \text { ss }) \text { : } \\
& >\text { summand: }=\operatorname{subs}(i=n-j-k \text {, summand }) \text { : } \\
& >\text { result:=sumtools [sumtohyper] (summand, } j \text { ): } \\
& >\text { result:=convert (result, binomial); }
\end{aligned}
$$

result $:=y^{k} z^{(n+1)} \operatorname{binomial}(1+k+n, 1+2 k)$

$\operatorname{hypergeom}\left(\left[\frac{1}{2}+k, n+k+2, k,-n+k\right],\left[k+1,1+2 k, \frac{3}{2}+k\right], y\right)$

Therefore, we have finally computed that

$$
\begin{gathered}
\tau_{k}^{n}(t)=e^{-k t}\left(\begin{array}{c}
1+k+n \\
1+2 k
\end{array}\right) . \\
\left.{ }_{4} F_{3}\left(\begin{array}{c}
\frac{1}{2}+k, n+k+2, k,-n+k \\
k+1,1+2 k, \frac{3}{2}+k
\end{array}\right) e^{-t}\right) .
\end{gathered}
$$

By taking derivative w.r.t $t$, this representation of $\tau_{k}^{n}$ as ${ }_{4} F_{3}$-hypergeometric function is easily seen to be equivalent to (10). Hence our computations have proved identity (9).

The plot

$$
\begin{aligned}
& >\text { plot (simplify( } \\
& >\operatorname{subs}(\mathrm{y}=(1-\mathrm{x}) / 2, \mathrm{z}=1, \mathrm{n}=30, \mathrm{k}=6, \mathrm{result})), \mathrm{x}=-1 \ldots 1) \text {; }
\end{aligned}
$$

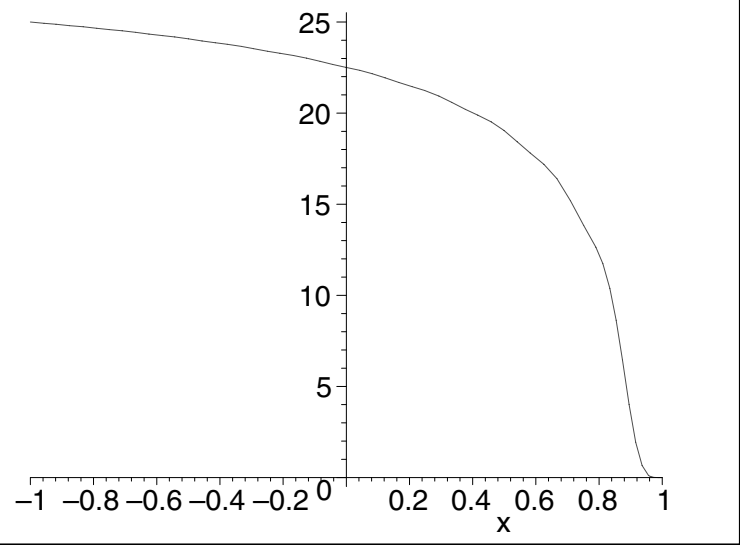


shows the nonnegativity of $\tau_{6}^{30}$. Morover it indicates that $\tau_{k}^{n}$ is much less oscillatory than its derivative.

\section{FURTHER RESULTS}

In this section we briefly mention some more examples for the use of Theorem 1 which, although not directly connected to the proof of the Bieberbach conjecture, still deal with the Löwner chain $w(z, y)$ of the Koebe function. These examples emphasize the rich structure of this function.

For this purpose we present some generating functions with hypergeometric representations, and leave the respective second application of the FPS algorithm to the reader:

- generating function $\left(\frac{1+w(z, y)}{1-w(z, y)}\right)^{m}$ :

$>\operatorname{standardsum}\left(\operatorname{FPS}\left(((1+w) /(1-w))^{\wedge} m, y, j\right)\right)$;

$$
\sum_{j=0}^{\infty} \frac{z^{j}\left(\frac{1}{(-1+z)^{2}}\right)^{j}(-1)^{j} 4^{j}\left(-\frac{m}{2}\right)_{j} y^{j}}{j !}
$$

- generating function $w(z, y)^{k} \frac{1-w(z, y)}{1+w(z, y)}$ :

$>\operatorname{standardsum}\left(y^{\wedge} k * \operatorname{FPS}((w / y) \wedge k *(1-w) /(1+w), y, j)\right)$;

$$
\sum_{j=0}^{\infty} \frac{y^{k}(-1)^{j} z^{(j+k)}\left(\frac{1}{(-1+z)^{2}}\right)^{(j+k)}(1+2 k)_{2 j} y^{j}}{(1+2 k)_{j} j !}
$$

- generating function $\frac{w(z, y)^{k}}{1-w(z, y)^{m}}$ :

$>\operatorname{standardsum}\left(y^{\wedge} k * \operatorname{FPS}\left((w / y)^{\wedge} k /(1-w)^{\wedge} m, y, j\right)\right)$;

$\sum_{j=0}^{\infty} \frac{y^{k}(-1)^{j} z^{(j+k)}\left(\frac{1}{(-1+z)^{2}}\right)^{(j+k)}(2 k-m)_{2 j} y^{j}}{(-m+2 k+1)_{j} j !}$

- generating function $\frac{w(z, y)^{k}}{1+w(z, y)} \frac{1}{1-w(z, y)^{m}}$ :

$>\operatorname{standardsum}\left(y^{\wedge} k * \operatorname{FPS}\left((w / y)^{\wedge} k /(1-w)^{\wedge} m /(1+w), y, j\right)\right)$;

$$
\sum_{j=0}^{\infty} \frac{y^{k}(-1)^{j} z^{(j+k)}\left(\frac{1}{(-1+z)^{2}}\right)^{(j+k)}(2 k-m)_{2 j} y^{j}}{(2 k-m)_{j} j !}
$$

\section{POSITIVITY AND STURM SEQUENCES}

When de Branges had found his function system $\tau_{k}^{n}(t)$, he was able to check the Bieberbach conjecture by hand computations for $n \leqq 6$. For this purpose he proved the nonpositivity of $\dot{\tau}_{k}^{n}(t)$ for $1 \leqq n \leqq 5,1 \leqq k \leqq n, t \geqq 0$.

He asked his colleague Walter Gautschi from Purdue University to verify (6) numerically, which was done for $n \leqq 30$, and de Branges became confident of the validity of the general statement (6).

Note, however, although Gautschi used Sturm sequences, because of the oscillatory nature of $\Lambda_{k}^{n}$ his numerical computations had to be very careful to obtain correct results.

Nowadays, we can apply Sturm sequences and count the roots easily by rational arithmetic to obtain correct countings since the input polynomials are elements of $\mathbb{Q}[y]$.

For example, the computation

$>\operatorname{sturm}($ simplify (

$>\operatorname{subs}(\mathrm{y}=(1-\mathrm{x}) / 2, \mathrm{z}=1, \mathrm{n}=30, \mathrm{k}=6$, result 1$)), \mathrm{x},-1,1)$; 1

proves that $\Lambda_{6}^{30}(x)$ has exactly one zero in the semi-open interval $(-1,1]$ (namely at the point $x=1$ ), and since its value at $x=-1$ is positive, $\Lambda_{6}^{30}(x)$ is nonnegative in $[-1,1]$.
Using Mathematica's CountRoots command which is based on a more efficient approach than Sturm sequences [4], the author could show relations (6) for $n=100, k=1, \ldots, 100$, e.g., hence Bieberbach's conjecture for $n=101$, within 110 seconds (Intel Pentium III, $1066 \mathrm{MHz}$ under Windows 2000).

\section{CONCLUSION}

In this article, we show how the algorithmic computation of power series can be applied to generating functions that appear naturally in the study of the Koebe function, to obtain hypergeometric representations for the de Branges and Weinstein functions. Moreover, we have considered how algebraic computation enables the fast verification of the positivity results in the proofs of de Branges and Weinstein for fixed $n$ and $k$ using Sturm sequences or similar approaches.

\section{REMARKS}

The Maple packages FPS.mpl and hsum6.mpl can be downloaded from the author's web site http: /www . mathematik. uni-kassel.de/ ${ }^{k}$ koepf/Publikationen, where also a corresponding Maple Help Database is available.

\section{REFERENCES}

[1] Askey, R. and Gasper, G.: Positive Jacobi polynomial sums II. Amer. J. Math. 98, 1976, 709-737.

[2] Bieberbach, L.: Über die Koeffizienten derjenigen Potenzreihen, welche eine schlichte Abbildung des Einheitskreises vermitteln. Semesterberichte Preuss. Akad. Wiss. 38, 1916, 940-955.

[3] De Branges, L.: A proof of the Bieberbach conjecture. Acta Math. 154, 1985, 137-152.

[4] Collins, G. E. and Krandick, W.: An efficient algorithm for infallible polynomial complex roots isolation. In: Wang, Paul S. (Ed.): Proceedings of ISSAC'92, 1992, 189-194.

[5] Ekhad, S. B. and Zeilberger, D.: A high school algebra, "formal calculus", proof of the Bieberbach conjecture [after L. Weinstein]. Jerusalem Combinatorics'93, Contemp. Math. 178, Amer. Math. Soc., Providence, 1994, 113-115. Review in Zentralblatt für Mathematik 0894.30013,

[6] Gruntz, D. and Koepf, W.: Maple package on formal power series. Maple Technical Newsletter 2 (2), 1995, $22-28$.

[7] Gruntz, D., Koepf, W. and Müller, D.: FPS.mpl for Maple 8, http: /www .mathematik.uni-kassel.de/ roepf/Publikationen, 2002.

[8] Koepf, W.: Power series in computer algebra. Journal of Symbolic Computation 13, 1992, 581-603.

[9] Koepf, W.: Algorithms for $m$-fold hypergeometric summation. Journal of Symbolic Computation 20, 1995, 399-417.

[10] Koepf, W.: Hypergeometric Summation. Vieweg, Braunschweig/Wiesbaden, 1998.

[11] Koepf, W. and Schmersau, D.: On the de Branges Theorem. Complex Variables 31, 1996, 213-230.

[12] Koepf, W. and Schmersau, D.: Weinstein's functions and the Askey-Gasper identity. Integral Transforms and Special Functions 5, 1997, 227-246.

[13] Lebedev, N. A. and Milin, I. M.: An inequality. Vestnik Leningrad Univ. 20, 1965, 157-158 (Russian). 
[14] Löwner, K.: Untersuchungen über schlichte konforme Abbildungen des Einheitskreises I. Math. Ann. 89, 1923, 103-121.

[15] Todorov, P.: A simple proof of the Bieberbach conjecture. Bull. Cl. Sci., VI. Sér., Acad. R. Belg. 3 (12), 1992, 335-346.

[16] Weinstein, L.: International Mathematics Research Notices 5, 1991, 61-64.
[17] Wilf, H.: A footnote on two proofs of the Bieberbach-de Branges Theorem. Bull. London Math. Soc. 26, 1994, 61-63.

[18] Wilf, H. and Zeilberger, D.: An algorithmic proof theory for hypergeometric (ordinary and " $q$ ") multisum/integral identities. Invent. Math. 103, 1992, 575-633. 